\title{
Tick-borne haemoparasites in African buffalo (Syncerus caffer) from two wildlife areas in Northern Botswana
}

Dewald Eygelaar ${ }^{1}$, Ferran Jori ${ }^{2,3,5}$, Mokganedi Mokopasetso 4,6, Kgomotso P Sibeko ${ }^{1}$, Nicola E Collins ${ }^{1}$, Ilse Vorster ${ }^{1}$, Milana Troskie ${ }^{1}$ and Marinda C Oosthuizen ${ }^{*^{*}}$

\begin{abstract}
Background: The African buffalo (Syncerus caffer) is a host for many pathogens known to cause economically important diseases and is often considered an important reservoir for livestock diseases. Theileriosis, heartwater, babesiosis and anaplasmosis are considered the most important tick-borne diseases of livestock in sub-Saharan Africa, resulting in extensive economic losses to livestock farmers in endemic areas. Information on the distribution of tick-borne diseases and ticks is scarce in Northern Botswana. Nevertheless, this data is necessary for targeting surveillance and control measures in livestock production at national level.

Methods: In order to address this gap, we analyzed 120 blood samples from buffalo herds for the presence of common tick-borne haemoparasites causing disease in livestock, collected in two of the main wildlife areas of Northern Botswana: the Chobe National Park (CNP, $n=64)$ and the Okavango Delta $(\mathrm{OD}, \mathrm{n}=56)$.

Results: Analysis of the reverse line blot (RLB) hybridization assay results revealed the presence of Theileria, Babesia, Anaplasma and Ehrlichia species, either as single or mixed infections. Among the Theileria spp. present, T. parva (60\%) and T. mutans (37\%) were the most prevalent. Other species of interest were Anaplasma marginale subsp. centrale (30\%), A. marginale (20\%), Babesia occultans (23\%) and Ehrlichia ruminantium (6\%). The indirect fluorescent antibody test (IFAT) indicated $74 \%$ of samples to be positive for the presence of T. parva antibodies. Quantitative real-time PCR (qPCR) detected the highest level of animals infected with T. parva ( $81 \%$ of the samples). The level of agreement between the tests for detection of T. parva positive animals was higher between QPCR and IFAT (kappa $=0.56)$, than between $\mathrm{qPCR}$ and RLB $(\mathrm{kappa}=0.26)$ or the latter and IFAT (kappa $=0.15)$.

Conclusions: This is the first report of tick-borne haemoparasites in African buffalo from northern Botswana, where animals from the CNP showed higher levels of infection than those from OD. Considering the absence of fences separating wildlife and livestock in the CNP and the higher levels of some parasite species in buffalo from that area, surveillance of tick-borne diseases in livestock at the interface in the CNP should be prioritized.
\end{abstract}

Keywords: Botswana, African buffalo, Haemoparasites, Tick-borne diseases, Theileria, Babesia, Anaplasma, Ehrlichia, Reverse line blot hybridization assay, Real-time PCR, IFAT

\footnotetext{
* Correspondence: Marinda.Oosthuizen@up.ac.za

${ }^{1}$ Department of Veterinary Tropical Diseases, Faculty of Veterinary Science, University of Pretoria, Private Bag X04, Onderstepoort 0110, South Africa Full list of author information is available at the end of the article
} 


\section{Background}

Theileriosis, babesiosis, anaplasmosis and heartwater are considered to be the most important tick-borne diseases (TBDs) of livestock in sub-Saharan Africa, resulting in extensive economic losses to farmers in endemic areas. The African buffalo (Syncerus caffer) is the natural reservoir host of Theileria parva, which is transmitted by the tick species, Rhipicephalus appendiculatus, $R$. zambeziensis and $R$. duttoni [1,2]. T. parva causes East Coast fever (ECF), which occurs in eastern and central Africa. ECF was introduced into southern Africa in the early 1900s through cattle importation from East Africa and was eradicated from South Africa in the 1950s [3,4]. T. parva also causes Corridor Disease, which is still prevalent in South Africa in areas where buffalo and cattle share grazing grounds in the presence of its tick vectors. It is a controlled disease in South Africa because of a concern that ECF might recur $[5,6]$. Although $T$. parva distributions have been described in Mozambique [7], Zambia [8] and Zimbabwe [3], no information on the distribution of T. parva is available for many other southern African countries, including Botswana.

In addition to $T$. parva, buffalo are also thought to be the original reservoir host of other non-pathogenic, mildly pathogenic and benign Theileria species namely, Theileria mutans, Theileria velifera, Theileria buffeli, Theileria sp. (buffalo) [9,10] and Theileria sp. (bougasvlei) [11,12]. Theileria parasites usually occur as mixed infections in buffalo and cattle. Although the benign and nonpathogenic forms do not have any significant economic importance, their presence could interfere with the interpretation of results obtained in some diagnostic tests designed to diagnose the pathogenic T. parva. Diagnostic tests for $T$. parva include microscopic examination of blood smears for the presence of piroplasms and schizonts, and serological methods such as the indirect fluorescent antibody test (IFAT) which is routinely used in South Africa for T. parva antibody detection in "disease free" buffalo [13,14]. Molecular diagnostic methods detect specific parasite sequences in DNA extracts from blood or tissue samples. The reverse line blot (RLB) hybridization assay makes use of polymerase chain reaction (PCR) amplification of haemoparasite small subunit ribosomal RNA genes (srRNA) which are screened with group- and species-specific probes for the simultaneous detection and identification of haemoparasites in mixed infections [15]. To date, the most sensitive molecular test for the detection of $T$. parva is a quantitative real-time PCR (qPCR) using hybridization probe chemistry, where the central region of the parasite $18 \mathrm{~S}$ rRNA gene is amplified and the presence of $T$. parva is confirmed by melting curve analysis [16]. It is currently routinely used to test for T. parva infections in buffalo and cattle in South Africa as part of the Corridor disease control strategy.
It has been shown that African buffalo are also carriers of a number of other tick-borne parasites which are detrimental to livestock including Ehrlichia ruminantium, Babesia bigemina, B. bovis, Anaplasma marginale and A. marginale subsp. centrale [17-19]. Although buffalo show no disease symptoms, as reservoir hosts, they may represent a threat to the livestock industry. Ehrlichia ruminantium, an intracellular rickettsial bacterium, is the causative agent of heartwater (cowdriosis) and is transmitted by three-host ticks belonging to the genus, Amblyomma [20]. Babesia bigemina and B. bovis cause bovine babesiosis, commonly known as redwater fever. Tick vectors for these parasites include Rhipicephalus microplus (formerly Boophilus microplus) and Rhipicephalus annulatus (formerly Boophilus annulatus). It is believed that Babesia is the second most common blood parasite after trypanosomes representing a significant health risk for cattle [21]. Anaplasma marginale causes bovine anaplasmosis which is characterized by the infiltration of the host's red blood cells. It can be transmitted to other hosts through mechanical transmission but the most important mode of transmission is via tick bites, the main tick vector being $R$. decoloratus (formerly Boophilus decoloratus) [22,23]. Anaplasma marginale subsp. centrale causes a milder form of anaplasmosis, and is used in a live blood vaccine in many countries, including South Africa [24].

Generally speaking, publications on significant tickborne haemoparasites in Botswana are limited [25-28] and there are no published reports of the presence of $T$. parva in livestock in this country. In addition, published literature on the occurrence of pathogens in buffalo populations from Botswana is very scarce [29]. Therefore, the goal of this study was to determine the prevalence of tick-borne parasites circulating in two distinct buffalo populations from Northern Botswana using different diagnostic methods and to use these data to compare the performance of those tests in detecting $T$. parva in buffalo.

\section{Methods}

\section{Buffalo sampling}

The Chobe National Park (CNP) and Okavango Delta (OD) are located in two different districts of Northern Botswana (Chobe and Ngamiland Districts, respectively) and represent the largest wildlife areas in this part of the country. They are both integrated in the Foot and Mouth Disease infected area, a large part of the northern region of Botswana devoted to wildlife conservation in which buffalo populations are separated from the primary cattle export and buffer zones by the use of veterinary cordon fences (Figure 1). The Chobe, Zambezi and Okavango rivers are the largest in the region, providing abundant water throughout the year. Rainfall is strongly 


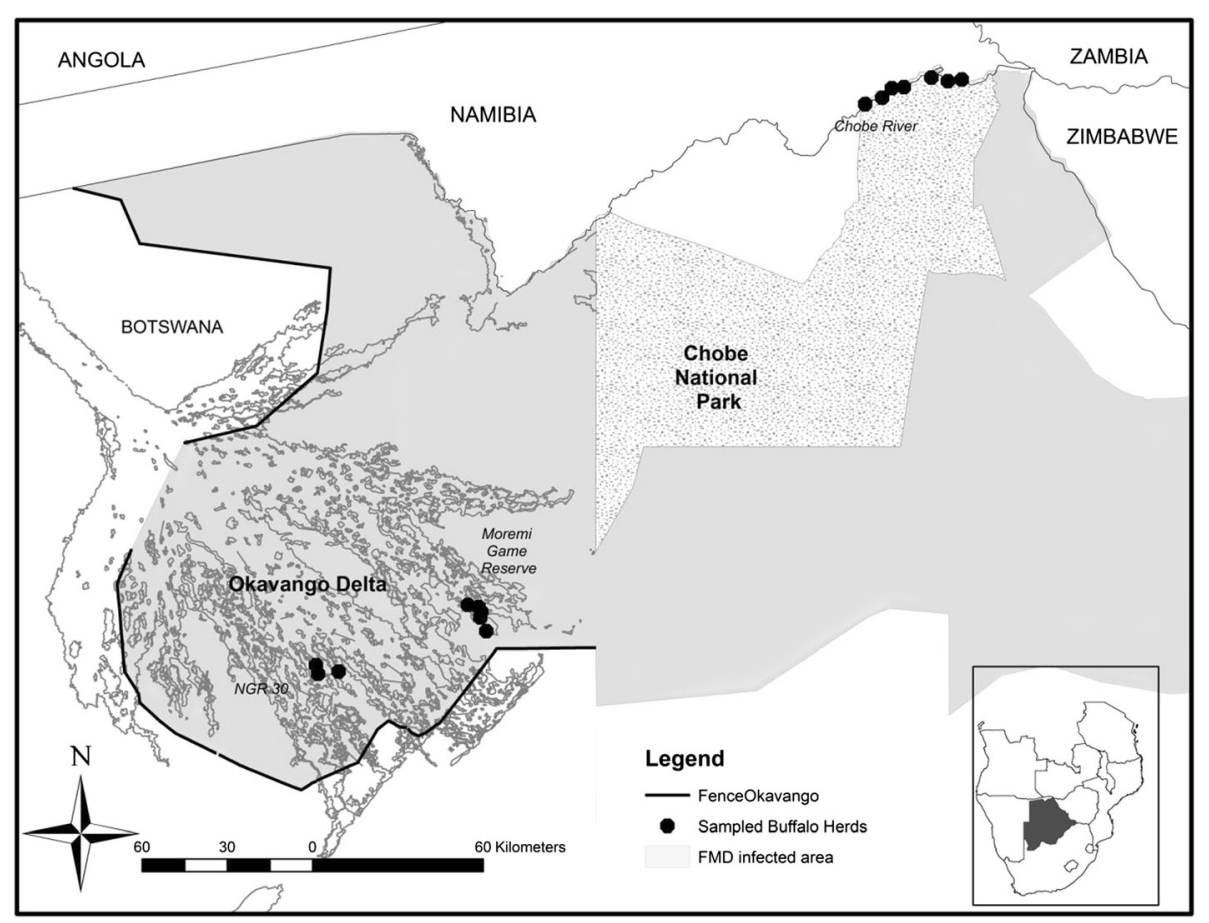

Figure 1 Areas where samples were collected from buffalo herds in northern Botswana. The three distinct areas where buffalo captures took place are indicated in italic characters. Individual capture sites are indicated by black dots.

seasonal, occurring mostly from December to April (wet season). Vegetation consists mainly of deciduous dry woodland and scattered grasslands. Wildlife abundance is fundamentally dependent on rainfall and water availability and varies cyclically throughout the years [30]. The CNP encompasses $10700 \mathrm{~km}^{2}$ of savannah grassland. The boundaries of CNP are natural, the Chobe river in the north constituting the natural border between Botswana and Namibia. There is no physical barrier preventing contacts between cattle and wildlife and the main water source for the animals in that area is the Chobe river which is exposed to seasonal variations of water levels. The OD encompasses $16000 \mathrm{~km}^{2}$ and contrary to the CNP, it is delineated from livestock areas by a double veterinary cordon fence to prevent contacts between wildlife and cattle [29], and it is largely flooded throughout the year. According to the last available wildlife census from Northern Botswana, buffalo populations and densities are estimated at 31500 individuals and 0.94 individuals $/ \mathrm{km}^{2}$ in OD and 7500 individuals and 0.23 individuals $/ \mathrm{km}^{2}$ in the CNP [31].

The sampling process was opportunistic and details of the capture approach were described [29]. During the capture process, blood samples were collected from a total of 120 individual buffalo. In the CNP, buffalo were captured along the Chobe river and blood samples were collected from 64 individual buffalo belonging to seven distinct herds. In the OD, 8 buffalo herds were sampled in two different management units: the Moremi Game Reserve (MGR) ( $\mathrm{n}=18$ individuals, 4 herds) and in the NG30 area ( $\mathrm{n}=38$ individuals, 4 herds) (Figure 1). Buffalo densities in those locations were estimated at 1.88 buffalo $/ \mathrm{km}^{2}$ for the Chobe river, 1.37 buffalo $/ \mathrm{km}^{2}$ for the MGR and 3.55 buffalo $/ \mathrm{km}^{2}$ for the NG30 area of the OD (31). The sex and age of the animals were recorded. Age was measured according to dentition; animals younger than 3 years were considered as young and animals older than 3 years were considered to be adults. Whole blood samples were obtained from the jugular vein, maintained in refrigeration and sent to the Botswana National Veterinary Laboratory in Gaborone. There, they were centrifuged at $1500 \mathrm{xg}$ for 15 minutes. Sera was then harvested with a pipette and stored frozen at $-20^{\circ} \mathrm{C}$, until the samples were ready to be sent to the Agricultural Research Council-Onderstepoort Veterinary Institute (ARC-OVI) in South Africa for analysis.

\section{Blood smears and DNA extraction}

A total of 120 thin layer smears were prepared in the field from a blood drop of the ear sublime vein of the captured buffalo (two individuals were missed). Those were dried in the sun and fixed with methanol. At the laboratory, each smear was stained with Giemsa dye following the standard procedures. Genomic DNA was extracted from the Giemsa-stained slides using the QIAmp DNA mini kit (Qiagen) following the manufacturer's 
protocol. The DNA was eluted into $100 \mu \mathrm{l}$ of TE buffer and stored at $-20^{\circ} \mathrm{C}$ until further use.

\section{PCR amplification and reverse line blot (RLB) hybridization assay}

A total of 120 DNA samples were tested using the RLB hybridization assay as previously described [15,7]. Briefly, the V4 hypervariable region of the parasite 18S rRNA gene was amplified using primers RLB-F2 and RLB-R2 [32], while the V1 region of the parasite 16S rRNA gene was amplified from Ehrlichia and Anaplasma species using primers Ehr-F and Ehr-R [33]. The PCR reaction was prepared as follows: $5 \mu$ DNA (30-50 ng), $12.5 \mu$ l Platinum Quantitative PCR SuperMix-UDG (Invitrogen, The Scientific Group, South Africa), and 20 pmol of each primer made up to a total volume of $25 \mu \mathrm{l}$ using nuclease-free water. Amplification was done using a touchdown PCR programme as previously described [32]. A T. parva positive buffalo DNA sample, 102 [16], and nuclease-free water were used as positive and negative controls, respectively. Amplicons were visualised on a $2 \%$ ethidium bromide-stained agarose gel and then screened by the RLB hybridization assay as previously described [15,34]. The Theileria, Ehrlichia, Anaplasma and Babesia group- and species-specific oligonucleotide probes used were the same as those described in [35] and [36].

\section{Indirect fluorescent antibody test (IFAT)}

After discarding haemolysed samples, only a total of 108 serum samples collected from buffalo were available to be tested using the IFAT $[13,37,38]$ according to OIE standards [39]. The test was conducted at the ARC-OVI using two dilutions, $1 / 40$ and $1 / 80$. The presence of fluorescence in both the $1 / 40$ and $1 / 80$ dilutions was considered as a positive result in serum from buffalo, indicative of the presence of T. parva antibodies (Olivier Matthee, personal communication).

\section{T. parva-specific quantitative real-time $P C R$ ( $q P C R$ )}

Among the 120 smears collected, one sample was discarded because of an insufficient amount of DNA for the test. A total of 119 DNA samples were subjected to the $T$. parva-specific qPCR assay as previously described [16]. Briefly, the T. parva-specific forward and Theileria genus-specific reverse primers [16] were used to amplify a $167 \mathrm{bp}$ fragment of the parasite V4 variable region of the $18 \mathrm{~S}$ rRNA gene. For the specific detection of T. parva amplicons, the hybridization probes T. parva anchor and T. parva sensor (640 nm LC Red) were included in the PCR reaction which consisted of $4 \mu$ of $10 \times$ LightCyclerFastStart DNA Master ${ }^{\text {PLUS }}$ Hybridization Probes mix (with $2 \times$ final concentration), $0.5 \mathrm{mM}$ of each primer, $0.1 \mathrm{mM}$ of each hybridization probe, $0.5 \mathrm{U}$ Uracil-deoxy- glycosylase (UDG) and $4 \mu \mathrm{l}$ of the template DNA (30$50 \mathrm{ng}$ ) with a final volume of $20 \mu \mathrm{l}$. A T. parva positive buffalo DNA sample, 102 [16], and nuclease-free water were used as positive and negative controls, respectively. Amplification and melting curve analysis were done as previously described [16] in a LightCycler1 v2 (Roche Diagnostics, Mannheim, Germany). Fluorescence values were measured at $640 \mathrm{~nm}$.

\section{Statistical analysis}

Descriptive epidemiological measures were analyzed using Epi-Info software (CDC, Atlanta, USA) and were reported as percentages of positive animals to the different diagnostic tests. Chi square test calculations for homogeneity of two populations (Fischer exact test) were used to statistically evaluate the potential influence of age, sex, location and density of buffalo at the capture sites on the observed parasite prevalence. When the variance of the two groups was not homogenous, the Kruskall Wallis test was used. Values of $\mathrm{p}<0.05$ were considered significant. Agreement between the different diagnostic tests assessing the presence of $T$. parva or its antibodies (IFAT, qPCR and RLB) was calculated for those sera having a common result to those tests. Two by two comparisons of the results were expressed using the kappa value. Kappa is a widely used measure of test agreement defined as the quotient of the observed proportion of agreement beyond chance and the maximal proportion of agreement beyond chance [40]. A kappa of 0 indicates no agreement beyond chance, while a kappa of 1 indicates perfect agreement. A kappa of 0.5 indicates a moderate level of agreement.

\section{Ethical statement}

The study (Project nr. V082-12) was approved by the University of Pretoria Animal Ethics committee.

\section{Results \\ RLB}

The RLB results (Table 1) indicated the presence of Theileria, Babesia, Anaplasma and Ehrlichia species, either as single or as mixed infections in the buffalo populations from two wildlife areas assessed in northern Botswana. From a total of 120 blood smear samples tested, 23 samples (19.2\%) contained single infections while $80(66.7 \%)$ contained mixed infections. The most prevalent haemoparasite in the CNP was T. mutans (60.9\%) followed by T. parva (51.6\%), T. buffeli (46.9\%), B. occultans (40.6\%) and A. marginale subsp. centrale (31.3\%). In the OD, T. parva (69.6\%) was most prevalent followed by $A$. marginale subsp. centrale (28.6\%) and Theileria sp. (buffalo) (23.2\%).

RLB results indicated that a total of 72 of the 120 samples $(60.0 \%)$ tested positive for $T$. parva DNA. There 
Table 1 The occurrence of different haemoparasites in buffalo blood samples from two geographical areas in northern Botswana as determined by the RLB hybridization assay

\begin{tabular}{|c|c|c|c|}
\hline & Chobe National Park $(n=64)$ & Okavango delta $(n=56)$ & Total $(n=120)$ \\
\hline Single infections: & $5(7.8 \%)$ & $18(32.1 \%)$ & $23(19.2 \%)$ \\
\hline T. parva & $1(1.6 \%)$ & $15(26.8 \%)$ & $16(13.3 \%)$ \\
\hline T. mutans & $3(4.7 \%)$ & 0 & $3(2.5 \%)$ \\
\hline A. marginale subsp. centrale & 0 & $2(3.6 \%)$ & $2(1.7 \%)$ \\
\hline A. marginale & $1(1.6 \%)$ & $1(1.8 \%)$ & $2(1.7 \%)$ \\
\hline Mixed infections & $53(82.8 \%)$ & $27(48.2 \%)$ & $80(66.7 \%)$ \\
\hline T. parva & $32(50.0 \%)$ & $24(42.9 \%)$ & $56(46.6 \%)$ \\
\hline T. mutans & $36(56.3 \%)$ & $5(8.9 \%)$ & $41(34.2 \%)$ \\
\hline A. marginale subsp. centrale & $20(31.3 \%)$ & $14(25.0 \%)$ & $34(28.3 \%)$ \\
\hline T. buffeli & $30(46.9 \%)$ & $4(7.1 \%)$ & $34(28.3 \%)$ \\
\hline B. occultans & $26(40.6 \%)$ & $2(3.6 \%)$ & $28(23.3 \%)$ \\
\hline Theileria sp. (sable) & $25(39.1 \%)$ & $2(3.6 \%)$ & $27(22.5 \%)$ \\
\hline A. marginale & $13(20.3 \%)$ & $9(16.1 \%)$ & $22(18.3 \%)$ \\
\hline Theileria sp. (buffalo) & $8(12.5 \%)$ & $13(23.2 \%)$ & $21(17.5 \%)$ \\
\hline T. velifera & $9(14.1 \%)$ & $1(1.8 \%)$ & $10(8.3 \%)$ \\
\hline E. ruminantium & $4(6.3 \%)$ & $3(5.4 \%)$ & $7(5.8 \%)$ \\
\hline T. ovis & $3(4.7 \%)$ & $1(1.8 \%)$ & $4(3.3 \%)$ \\
\hline Anaplasma sp. Omatjenne & $1(1.6 \%)$ & $2(3.6 \%)$ & $3(2.5 \%)$ \\
\hline B. bovis & 0 & $2(3.6 \%)$ & $2(1.7 \%)$ \\
\hline Theileria/Babesia genus-specific only & $2(3.1 \%)$ & $5(8.9 \%)$ & $7(5.8 \%)$ \\
\hline Anaplasma/Ehrlichia genus-specific only & $1(1.6 \%)$ & $3(5.4 \%)$ & $4(3.3 \%)$ \\
\hline Negative/below detection limit & $2(3.1 \%)$ & $1(1.8 \%)$ & $3(2.5 \%)$ \\
\hline
\end{tabular}

was a significant difference $(\mathrm{p}=0.042)$ in the prevalence of $T$. parva-positive samples between the two wildlife areas. However, there was no significant association between prevalence of $T$. parva, and sex or age of the sampled animals (Table 2).

RLB results also showed significant differences in the prevalence of T. mutans, T. buffeli, B. occultans, Theileria sp. (sable) and $T$. velifera infections per wildlife area (Table 3). There was a significant difference $(\mathrm{p} \leq 0.05)$ between the age of animals that harboured $A$. marginale

Table 2 Comparison of prevalence of $T$. parva per location, age and sex with the three different tests performed

\begin{tabular}{lllll}
\hline Parameter & & RLB & IFAT & qPCR \\
\hline Wildlife area & CNP & $\mathbf{3 3 / 6 4}(\mathbf{5 1 . 6 \% )}$ & $\mathbf{3 9 / 6 0}(\mathbf{6 5 . 0 \% )}$ & $49 / 63(77.8 \%)$ \\
& OD & $\mathbf{3 9 / 5 6}(\mathbf{6 9 . 6 \% )}$ & $\mathbf{4 1 / 4 8}(\mathbf{8 5 . 4 \% )}$ & $\mathbf{4 7 / 5 6 ( 8 3 . 9 \% )}$ \\
\multirow{2}{*}{ Age } & Young & $\mathbf{1 9 / 3 8 ( 5 0 . 0 \% )}$ & $\mathbf{2 6 / 3 6 ( 7 2 . 2 \% )}$ & $27 / 38(71.1 \%)$ \\
& Adult & $48 / 77(62.3 \%)$ & $54 / 71(76.1 \%)$ & $66 / 77(85.7 \%)$ \\
Sex & Male & 23/45(51.1\%) & $31 / 43(72.1 \%)$ & $37 / 45(82.2 \%)$ \\
& Female & $44 / 70(62.9 \%)$ & $49 / 64(76.6 \%)$ & $57 / 70(81.4 \%)$ \\
\hline
\end{tabular}

Boldfaced values indicate a significant difference between test results for a given parameter $(\mathrm{p} \leq 0.05)$. subsp. centrale, T. buffeli, B. occultans and A. marginale infections (Table 3 ). There was a significant association $(\mathrm{p} \leq 0.05)$ between sex and the buffalo that tested positive for A. marginale subsp. centrale and T. velifera DNA (Table 3 ).

When comparing the T. parva RLB results in the three buffalo capture sites, $T$. parva prevalence was highest in the NG30 samples (76.3\%) followed by the MGR (55.6\%) samples and the CNP samples (51.6\%). Differences in $T$. parva prevalence as determined by RLB were only significant when comparing NG30 versus CNP ( $\mathrm{p}=0.01$ ) (Figure 2). There were no significant differences between the prevalence of other haemoparasites in the different capture sites or the numbers were too small to assess statistical differences

\section{IFAT}

T. parva antibodies were detected in 80 of the 108 (74.1\%) samples tested (Table 2). Of these, 40 (37.0\%) samples tested positive at $1 / 80$ and $40(37.0 \%)$ at $1 / 40$. More buffalo from OD (85.4\%) than from CNP (65.0\%) were seropositive for $T$. parva (Table 2) and this difference was significant $(\mathrm{p}=0.016)$. There was no significant difference between the age and sex of the animals that 
Table 3 Comparison of occurrence of other haemoparasites per wildlife area, sex and age as determined by the RLB hybridization assay

\begin{tabular}{|c|c|c|c|c|c|c|c|c|c|c|c|c|c|}
\hline & & T. mutans & $\begin{array}{l}\text { A. marginale ss } \\
\text { centrale }\end{array}$ & T. buffeli & B. occultans & $\begin{array}{l}\text { Theileria sp. } \\
\text { (sable) }\end{array}$ & A. marginale & $\begin{array}{l}\text { Theileria. sp } \\
\text { (buffalo) }\end{array}$ & T. velifera & $\begin{array}{l}\text { E. } \\
\text { ruminantium }\end{array}$ & T. ovis & $\begin{array}{l}\text { Anaplasma sp. } \\
\text { Omatjenne }\end{array}$ & B. bovis \\
\hline \multirow[t]{2}{*}{ Area } & CNP & $39 / 64$ (60.9\%) & 20/64 (31.3\%) & $30 / 64(46.9 \%)$ & $26 / 64(40.6 \%)$ & 25/64 (39.1\%) & 14/64 (21.9\%) & $8 / 64$ (12.5\%) & 9/64 (14.1\%) & 4/64 (6.3\%) & $3 / 64(4.7 \%)$ & $1 / 64(1.6 \%)$ & $0 / 64(0.0 \%)$ \\
\hline & OD & $5 / 56(8.9 \%)$ & 16/56 (28.6\%) & 4/56 (7.1\%) & 2/56 (3.6\%) & 2/56 (3.6\%) & 10/56 (17.9\%) & 13/56 (23.2\%) & $1 / 56(5.4 \%)$ & $3 / 56(5.4 \%)$ & $1 / 56(1.8 \%)$ & 2/56 (3.6\%) & $2 / 56(3.6 \%)$ \\
\hline \multirow[t]{2}{*}{ Age } & Young & 16/38 (42.1\%) & 19/38 (50.0\%) & $6 / 38(15.8 \%)$ & $4 / 38$ (10.5\%) & 7/38 (18.4\%) & $15 / 38(39.5 \%)$ & 4/38 (10.5\%) & 2/38 (5.3\%) & 2/38 (5.3\%) & $2 / 38(5.3 \%)$ & 0/38 (0.0\%) & $1 / 38(2.6 \%)$ \\
\hline & Adult & 27/77 (35.1\%) & 16/77 (20.8\%) & 27/77 (35.1\%) & 24/77 (31.2\%) & 20/77 (26.0\%) & $8 / 77$ (10.4\%) & 15/77 (19.5\%) & 8/77 (10.4\%) & $5 / 77(6.5 \%)$ & $2 / 77(2.6 \%)$ & 3/77 (3.9\%) & 0/77 (0.0\%) \\
\hline \multirow[t]{2}{*}{ Sex } & Male & 21/45 (46.7\%) & 19/45 (42.2\%) & 16/45 (35.6\%) & 14/45 (31.1\%) & 13/45 (28.9\%) & 13/45 (28.9\%) & 10/45 (22.2\%) & $7 / 45$ (15.6\%) & 4/45 (8.9\%) & $3 / 45(6.7 \%)$ & $1 / 45(2.2 \%)$ & $1 / 45(2.2 \%)$ \\
\hline & Female & 22/70 (31.4\%) & 16/70 (22.9\%) & 17/70 (24.3\%) & $14 / 70(20.0 \%)$ & $14 / 70(20.0 \%)$ & 10/70 (14.3\%) & 9/70 (12.9\%) & $3 / 70(4.3 \%)$ & $3 / 70(4.3 \%)$ & $1 / 70(1.4 \%)$ & 2/70 (2.9\%) & $0 / 70(0.0 \%)$ \\
\hline
\end{tabular}

Boldfaced values indicate a significant difference between test results for a given parameter $(p \leq 0.05)$ 


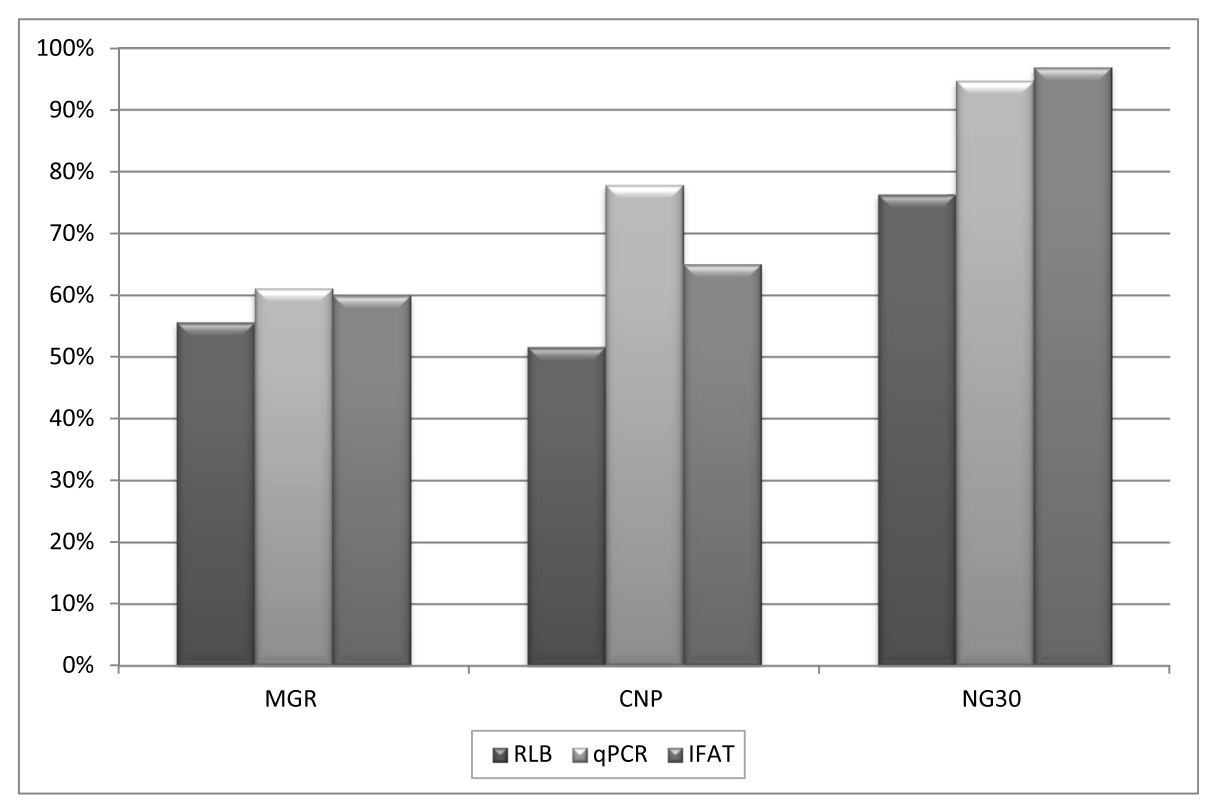

Figure 2 Pair wise comparison of capture location and prevalence of $T$. parva according to the different tests performed. Differences were significant ( $p \leq 0.05$ ) when comparing CNP and NG30 (for the 3 tests) and when comparing MGR and NG30 (for IFAT and qPCR). Note: buffalo densities in those locations were estimated at 1.88 buffalo/ $\mathrm{km}^{2}$ for the Chobe river, 1.37 buffalo/ $\mathrm{km}^{2}$ for the MGR and $3.55 \mathrm{buffalo/ \textrm {km } ^ { 2 }}$ for the NG30 area of the OD.

were seropositive (Table 2). Mean seroprevalence was higher (96.9\%) in the NG30 region than in the other two capture sites, $65.0 \%$ in the CNP and $60.0 \%$ in the MGR and those differences were highly significant. Differences in T. parva seroprevalence between MGR and CNP were not significant (Figure 2).

\section{Quantitative real-time PCR (qPCR)}

Melting curve analysis (Figure 3) confirmed the presence of T. parva DNA in 96 of 119 (80.7\%) samples tested. There were no significant differences between prevalence of T. parva with this test, and the wildlife area, age or sex of the animals (Table 2), but some significant differences were found when comparing capture sites. Mean prevalence of $T$. parva as determined by qPCR was higher in the NG30 region $(94.7 \%)$ than in the other two capture sites; $77.8 \%$ in the CNP and $61.1 \%$ in the MGR, respectively (Figure 2). The differences in $T$. parva prevalence between MGR and the CNP were not significant but it became highly significant when comparing $T$. parva prevalence in NG30 with that in the other two areas.

\section{Comparison of tests}

For IFAT, 80/108 (74.1\%) samples tested positive for $T$. parva antibodies, while for the qPCR and the RLB hybridization assay, 96/119 (80.7\%) and 72/120 (60.0\%) of the samples tested positive, respectively. The Kappa value when comparing IFAT and qPCR indicated a moderate level of agreement (0.561), while the comparison

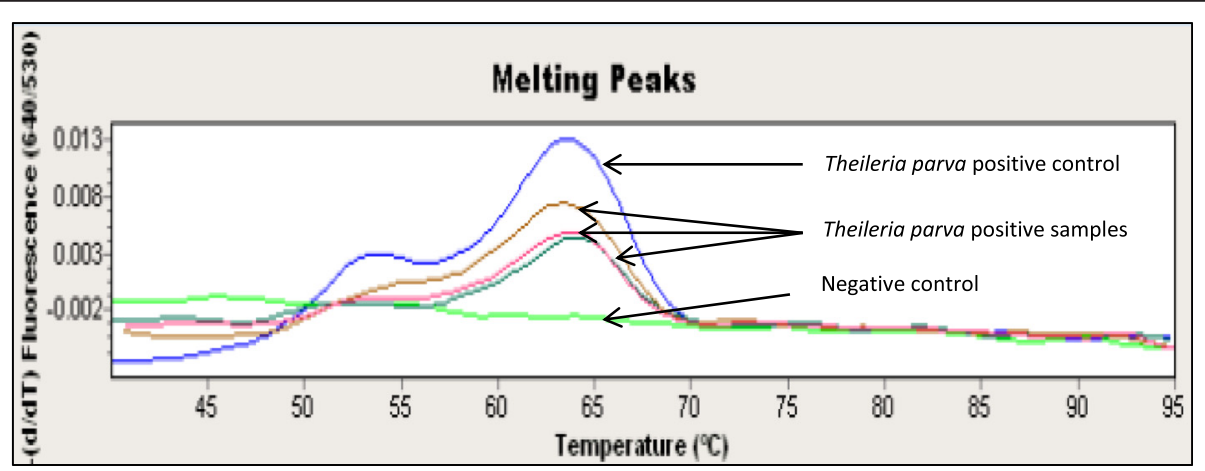

Figure 3 Representative melting curves at $\pm 63^{\circ} \mathrm{C}$ at $640 \mathrm{~nm}$ confirming the presence of $T$. parva positive samples. 
with RLB and the two other tests indicated a low level of agreement (Table 4). The observed prevalences of $T$. parva (RLB assay) and T. parva antibodies (IFAT) were both significantly higher in the OD than in CNP, while no significant difference was observed for the qPCR test.

\section{Discussion}

This is the first report on the presence of tick-borne haemoparasites in African buffalo from two of the most representative wildlife areas from northern Botswana. Our results provide new insights on the distribution of haemoparasites from buffalo transmissible to cattle across the two main wildlife areas in northern Botswana which can be inferred to the distribution of their specific vectors. Several important pathogenic haemoparasites which could present a constraint to the livestock industry in Botswana were identified. These included $T$. parva, A. marginale, B. bovis and E. ruminantium. A higher level of mixed infections was found in CNP compared to the locations sampled in the OD. This may be due to the fact that the Chobe river is the main source of water in the CNP and a high number of buffalo herds congregate along this water source during the dry season which might facilitate the exchange of ticks and their parasites between different individuals. In the OD, there is water all year round and despite some animal densities might be locally higher, buffalo herds might have less interactions between each other.

The RLB, IFAT and qPCR tests all indicated a high prevalence of $T$. parva presence or exposure in both CNP and OD. This indicates a high risk of spreading Corridor disease caused by $T$. parva from buffalo to cattle by the vector ticks at the livestock-wildlife interface. Other haemoparasites with high prevalence identified by the RLB included T. mutans, T. buffeli, B. occultans and Theileria sp. (sable) in CNP and $A$. marginale subsp. centrale, Theileria sp. (buffalo) and A. marginale in OD. Generally speaking, the buffalo population in the OD sample had lower levels of haemoparasite infections than the one in the CNP sample, with the exception of Theileria sp. (buffalo) and to a lesser extent Anaplasma sp. Omatjenne and B. bovis (in the two later cases, with very few positives were detected). In the specific case of $T$. parva, a significant association was observed between densities of buffalo in the capture location of the herds (only 3 measures of density were available) and prevalence

Table 4 Agreement expressed by kappa value when comparing diagnostic tests for Theileria parva two by two

\begin{tabular}{llll}
\hline Tests compared & Sample size & Kappa value & Standard error 95\% CI \\
\hline RLB vs qPCR & 119 & 0.256 & $0.09[0.09 ; 0.472]$ \\
IFAT vs qPCR & 107 & 0.561 & $0.096[0.3 ; 0.7]$ \\
IFAT vs RLB & 107 & 0.154 & $0.094[-0.031 ; 0.3]$ \\
\hline
\end{tabular}

found with the different tests, particularly in the case of the IFAT and qPCR results. This was more evident when comparing those areas where differences between density figures were more extreme (cf MGR and the NG30). However, buffalo density is only one possible cause of those differences, and many other habitat variations or ecological factors affecting host health or vector distribution and density in the different range areas where the buffalo herds were sampled, could also be responsible for those differences $[41,42]$. Therefore, further studies with a higher number of data and measures at the different herd locations would be necessary to detect explanatory factors accounting for those prevalence differences.

T. parva antibodies were detected in $74.1 \%$ of samples tested using the IFAT. Limitations of the IFAT include standardization of the test in buffalo samples, subjectivity towards the interpretation of results acquired and the difficulty of detecting low levels of parasite antibodies $[9,43]$. The IFAT is highly sensitive when testing for antibodies for only one species of Theileria, but in areas where different species overlap; cross-reactions between Theileria species are common (especially between $T$. parva, T. annulata and T. taurotragi) and reduce the specificity of the test $[9,40]$. However, the geographical distribution of $T$. annulata and $T$. parva does not overlap [44] and $T$. taurotragi was not identified in the Botswana buffalo samples. Therefore, the likelihood of cross-reactivity can be ruled out and our results most likely reflect the real presence and burden of $T$. parva, which would explain the high level of agreement between IFAT and qPCR. Another factor to take into account is the period elapsed from infection and the development of antibodies. With the use of schizont antigen, $T$. parva antibodies can first be detected 10 to 14 days post-infection and with piroplasm antigens 15 to 21 days post-infection. High levels of antibodies are still detectable 30 to 60 days after the animals have recovered from a $T$. parva infection which is followed by the gradual decrease of antibody levels. Antibodies can still be detected 4 to 6 months post-recovery and may persist for up to a year at such low levels that they may not be detected at a serum dilution of $1 / 40$ [39]. The animals in our study may have carried T. parva infections for a very long time as suggested by the very low antibody levels observed.

Comparing the efficiency of the different tests, we found that the qPCR (80.7\%) and IFAT (74.1\%) were far better in identifying $T$. parva positive samples than the RLB assay (60.0\%). Although there was correlation between the qPCR and RLB results in the detection of $T$. parva, minor differences between the results were observed; most notably in those samples with mixed haemoparasite infection. The qPCR assay can reliably detect $T$. parva in carrier animals with a piroplasm parasitaemia as 
low as $8.79 \times 10^{-4} \%$ [16]. The sensitivity of the RLB assay was determined at $10^{-6} \%$ parasitaemia, by testing serial dilutions of $T$. annulata-infected blood samples [15]. However, due to the likely presence of multiple parasites present in one sample, competition for available primers may occur in the PCR which in turn would lead to an underrepresentation of some of the parasites detected by RLB alone. Furthermore, weak hybridization and/or crossreactivity of probes may cause the RLB hybridization assay to yield less sensitive results than the qPCR and IFAT [45]. In addition, mixed infections could mask the presence of novel genotypes in the RLB assay and other tests would be needed to identify them $[46,47,15]$.

Risk factors influencing the prevalence of tick-borne parasites may include the distribution of tick vectors, the abundance of buffalo and cattle and their movement/migratory patterns, resistance of the hosts to the parasites and their tick vectors [48] and age of the host [42]. It has previously been found that older animals have a higher tick load than younger ones [42]. However, higher tick loads do not necessarily mean higher infection rates of haemoparasites. A model designed in East-Africa also determined that in wildlife-livestock interfaces where only cattle were treated with acaricides, $T$. parva remained a problem because this treatment had no effect on the disease transmission in buffalo. In addition, the continuous use of acaricides can have significant economic and ecological consequences [49]. Our study only identified a significant difference between age and infection rate for $B$. occultans and Theileria sp. (sable), which depending on the species of parasite, were higher in young (A. marginale, $A$. marginale subsp. centrale, ) or in adult animals ( $B$. occultans and Theileria buffeli). However, our sample was too small to be able to detect a consistent trend between age and infestation.

A number of studies have previously been conducted on buffalo in South Africa. In the Marakele National Park (MNP) and Kruger National Park (KNP) buffalo were tested for the presence of Theileria spp. using the RLB hybridization assay and the T. parva-specific qPCR assay [50]. The RLB results indicated the presence of $T$. parva, Theileria sp. (buffalo), T. mutans, T. buffeli and T. velifera in both parks. The qPCR assay identified 70\% of samples positive for $T$. parva and the RLB results indicated $40 \%$ of samples in both parks. In a separate study in the Hluhluwe-iMfolozi Park and the Greater Limpopo Transfrontier Park, T. parva, Theileria sp. (buffalo), T. mutans, T. buffeli and T. velifera were also identified in African buffalo [7]. In both studies, the T. parva-specific qPCR was found to be more sensitive than the RLB, correlating with the results found in our study. In another study done in the Hluhluwe-iMfolozi Park and Kruger National Park, Debiela [51] found the same Theileria spp. as in our study in Botswana, as well as A. marginale subsp. centrale, A. marginale, Anaplasma sp. Omatjenne, E. ruminantium and $B$. occultans.

In East Africa, in a study done in four different national parks in Uganda, buffalo were found to be carriers of T. parva, T. mutans, T. velifera, A. marginale and A. marginale subsp. centrale [52]. In two of these parks, buffalo also carried T. buffeli and Theileria sp. (buffalo). None of the animals sampled were carriers of T. taurotragi, B. bovis, B. bigemina, A. bovis or E. ruminantium. As in Uganda, the pathogenic $B$. bovis has previously been reported to be absent from buffalo in Botswana [53]. However, in the current study, we identified the parasite to be present in a low percentage of the OD buffalo tested. Similarly, E. ruminantium could be identified in a few CNP and OD buffalo tested. The significance of buffalo as possible reservoir host of some of these economically important haemoparasites (i.e. $A$. marginale, E. ruminantium) remains unknown.

Theileria sp. (sable), which is fatal to sable (Hippotragus niger) and roan antelope (Hippotragus equinus), but non-pathogenic to buffalo [34] was identified in some of the Botswana buffalo. However, it should be noted that the positive RLB signals might be due to cross reactions of the Theileria sp. (sable) probe with genotypes similar to Theileria sp. (sable) and/or with T. velifera and should be interpreted with caution [12]. Similarly, four samples tested positive for $T$. ovis which is usually found in goats and sheep. We can only speculate whether these are true findings due to incidental infections, or whether they are as a result of cross-reaction of the RLB probes with previously unknown targets or contamination with other target DNA.

The following important tick vectors have been identified in Botswana in previous studies: Amblyomma variegatum, Rhipicephalus decoloratus, R. zambeziensis, $R$. evertsi evertsi, $R$. simus, Hyalomma truncatum and $H$. marginatum rufipes [54-56]. These ticks are known to transmit most of the haemoparasites found in this study. These vectors may also be responsible for the transmission of T. buffeli and Theileria sp. (buffalo) but further research is needed to confirm this hypothesis, since the tick vectors of these parasite species remain unknown.

T. parva is known to occur in Zambia [8] and South Africa [57,58], but this is the first written report of its occurrence in northern Botswana, despite its presence has been suspected for several years. Currently, there are no regulations instituted for the systematic surveillance and control of tick-borne diseases in Botswana. In addition, Corridor Disease in cattle is fulminant and makes it difficult to detect clinical cases in live animals. Therefore, the present work emphasizes the role of the African buffalo, as a sentinel species to identify the presence and circulation of livestock pathogens. The 
presence of Rhipicephalus appendiculatus and East Coast fever in northern Botswana has been predicted in spatial risk models by some authors [59]. When infected buffalo share the same home ranges with cattle and other domestic animals, those haemoparasites can be transmitted to cattle through infected tick bites. This information on the circulation of TBD can contribute to raise awareness among veterinary officials and rural communities living at the wildlife-livestock interface so that control measures (prevention of wildlife-cattle contacts, regular dipping) can be implemented to mitigate their economic impact. In a recent comparative assessment of cattle herds in three different wildlife/livestock interfaces in Zimbabwe, significantly higher levels of $T$. parva antibodies were found in those areas that were unfenced when compared with those that had a physical separation between wildlife and livestock [60]. Therefore, we can hypothesize that this parasite is less likely to be transmitted from buffalo to cattle in the OD, due to the presence of a veterinary cordon fence preventing contacts with cattle and surrounding the game reserve. To the contrary in the CNP, where there is no physical separation between buffalo and cattle, transmission of common diseases from buffalo to cattle is likely to occur more frequently [29] and future surveillance efforts should be targeted in priority towards livestock from this area.

\section{Conclusions}

This paper illustrates the diversity of haemoparasites present in African buffalo from northern Botswana and highlights the role of African buffalo as a sentinel species for livestock tick-borne pathogens. Our results indicate the significance of the African buffalo as reservoir host for important tick-borne haemoparasites that can cause severe disease in cattle. They also suggest that qPCR and IFAT are more efficient in detecting T. parva exposed buffalo than the RLB test. These results should contribute to raise awareness among veterinary authorities regarding the potential occurrence of these parasites in cattle so that appropriate control and surveillance protocols taking into account the presence of infected wildlife reservoirs in those areas can be designed at the wildlife-livestock interface.

\section{Competing interests}

The authors declare that they have no competing interests.

\section{Authors' contributions}

$\mathrm{DE}$ carried out the molecular diagnostic assays and wrote the first draft of the manuscript. FJ conducted fieldwork for the collection of blood, performed the statistical analysis and interpreted the data. KPS provided GPCR training, while the training in DNA extraction and the RLB hybridization assay were provided by IV and MT. MCO supervised the laboratory work, interpreted the data and is the main supervisor of the student (DE). DE, FJ, NE and MCO arranged the data, formulated and prepared the manuscript. All authors read and approved the final manuscript.

\section{Acknowledgements}

This research was financially supported by United Nations Food and Agriculture Organization ECTAD Office in Gaborone (LoAPR 43231, New PR 45371) and the South African National Research Foundation (NRF, CSUR program: SUR2009062200001347). It also falls under the Belgian Directorate General for Development Co-operation Framework agreement ITM/DGCD. We thank the Department of Veterinary Services and the Department of National Parks and Wildlife from Botswana for facilitating and approving this investigation.

\section{Author details}

${ }^{1}$ Department of Veterinary Tropical Diseases, Faculty of Veterinary Science, University of Pretoria, Private Bag X04, Onderstepoort 0110, South Africa. ${ }^{2}$ Department of Zoology and Entomology, University of Pretoria, Pretoria 0002, South Africa. ${ }^{3}$ UPR AGIRs, CIRAD, Campus International de Baillarguet, Montpellier 34398, France. ${ }^{4}$ FAO-ECTAD Office for Southern Africa, Gaborone, Botswana. ${ }^{5}$ Department of Animal Science and Production, Botswana College of Agriculture, Private Bag 0027, Gaborone, Botswana. ${ }^{6}$ Botswana Vaccine Institute, Private Bag 0031, Gabarone, Botswana.

Received: 9 September 2014 Accepted: 29 December 2014

Published online: 15 January 2015

\section{References}

1. Norval RAl, Lawrence JA, Young AS, Perry BD, Dolan TT, Scott J. Theileria parva: influence of vector, parasite and host relationships on the epidemiology of theileriosis in Southern Africa. Parasitol. 1991;102:347-56.

2. Uilenberg G. Immunization against diseases caused by Theileria parva: a review. Trop Med Int Health. 1999;4:12-20.

3. Latif AA, Hove T. History and critical review of Theileria parva (Boleni), the vaccine stock against Zimbabwean cattle theileriosis. Ticks Tick Borne Dis. 2011;2:163-7.

4. Lawrence JA. The differential diagnosis of the bovine theileriosis of Southern Africa. J S Afr Vet Ass. 1979;50:311-3.

5. Potgieter FT, Stoltsz WH, Blouin EF, Roos JA. Corridor disease in South Africa: a review of the current status. Onderstepoort I S Afr Vet Assoc. 1988;59:155-60.

6. Stoltsz WH. Theileriosis in South Africa: a brief review. Rev - Off Int Epizoot. 1989;8:93-102.

7. Chaisi ME, Sibeko KP, Collins NE, Potgieter FT, Oosthuizen MC. Identification of Theileria parva and Theileria sp. (buffalo) $18 \mathrm{~S}$ rRNA gene sequence variants in the African Buffalo (Syncerus caffer) in Southern Africa. Vet Parasitol. 2011;182:150-62.

8. Munang'andu HM, Siamudaala V, Matandiko W, Mulumba M, Nambota A, Munyeme $M$, et al. Detection of Theileria parva antibodies in the African buffalo (Syncerus caffer) in the livestock-wildlife interface areas of Zambia. Vet Parasitol. 2009;166:163-6.

9. Norval RAI, Perry BD, Young AS. The Epidemiology of Theileriosis in Africa. London: Academic Press; 1992.

10. Stoltsz WH. Theileriosis in the African Buffalo. In: Proceedings of a Symposium on the African Buffalo as a Game Ranch Animal, Wildlife Group of the South African Veterinary Association. South Africa: Onderstepoort; 1996. p. 126-30.

11. Zweygarth E, Koekemoer O, Josemans Al, Rambritch N, Pienaar R, Putterill J, et al. Theileria-infected cell line from an African buffalo (Syncerus caffer). Parasitol Res. 2009;105:579-81.

12. Mans BJ, Pienaar R, Latif AA, Potgieter FT. Diversity in the $18 S$ SSU rRNA V4 hyper-variable region of Theileria spp. in Cape buffalo (Syncerus caffer) and cattle from southern Africa. Parasitol. 2011;138:766-79.

13. Schindler $R$, Mehlitz $D$, Matson B. Serological and immunological studies on Theileria lawrencei infection in cattle. Z Tropenmed Parasitol. 1969;20:162-83.

14. Laubscher $L$, Hoffman $L$. An overview of disease-free buffalo breeding projects with reference to the different systems used in South Africa. Sustainability. 2012;4:3124-40.

15. Gubbels JM, de Vos AP, van der Weide M, Viseras J, Schouls LM, de Vries E, et al. Simultaneous detection of bovine Theileria and Babesia species by reverse line blot hybridization. J Clin Microbiol. 1999;37:1782-9.

16. Sibeko KP, Oosthuizen MC, Collins NE, Geysen D, Rambritch NE, Latif AA, et al. Development and evaluation of a real-time polymerase chain reaction test for the detection of Theileria parva infections in Cape buffalo (Syncerus caffer) and cattle. Vet Parasitol. 2008;155:37-48. 
17. Andrew HR, Norval RAI. The carrier status of sheep, cattle and African buffalo recovered from heartwater. Vet Pathol. 1989:34:261-6.

18. Iseki H, Alhassan A, Ohta N, Thekisoe OMM, Yokoyama N, Inoue N, et al. Development of a multiplex loop-mediated isothermal amplification (mLAMP) method for the simultaneous detection of bovine Babesia parasites. J Microbiol Methods. 2007;71:281-7.

19. Kuttler KL. Serological survey of anaplasmosis incidence in East Africa, using the complement-fixation test. Bull Epizoot Dis Afr. 1965;13:257-62.

20. Allsopp BA. Natural history of Ehrlichia ruminantium. Vet Parasitol. 2010;167:123-35.

21. Hunfeld KP, Hildebrandt A, Gray JS. Babesiosis: recent insights into an ancient disease. Int J Parasitol. 2008;38:1219-37.

22. Potgieter FT, van Rensburg $\sqcup$. Tick transmission of Anaplasma centrale. Onderstepoort J Vet Res. 1987;54:5-7.

23. Potgieter FT, Stoltsz WH. Bovine Anaplasmosis. In: Coetzer JAW, Tustin RC, editors. Infectious Diseases of Livestock, vol. 1. 2nd ed. Oxford: University Press; 2004. p. 594-616.

24. Potgieter FT, van Rensburg LJ. Infectivity, virulence and immunogenicity of Anaplasma centrale live blood vaccine. Onderstepoort J Vet Res. 1983:47:285-6.

25. Carmichael $\mathrm{H}$, Hobday E. Blood parasites of some wild bovidae in Botswana. Onderstepoort J Vet Res. 1975;42:55-62.

26. Sharma SP, Losho TC, Baipoledi EK, Nyange J. The prevalence of heartwater in domestic ruminants in Botswana. Bull Anim Health Prod Afr. 2003;51:215-21.

27. Batisani N, Waugh E, Mothubane O, Akanyang L. The geographical prevalence and potential epidemiology of heartwater in Botswana: implications for planning control under climate change. Bots J Agric Appl Sci. 2012;8:82-100.

28. Mahabile M. Measuring transaction costs in marketing cattle in Southern Botswana. Bots J Agric Appl Sci. 2013;9:2.

29. Jori F, Mokospasetso M, Etter E, Munstermann S, Newman SH, Michel A. Preliminary assessment of bovine tuberculosis at the livestock/wildlife interface in two protected areas of Northern Botswana. Transbound Emerg Dis. 2013;60:28-36.

30. Alexander KA, Blackburn JK, Vandewalle ME, Pesapane R, Baipoledi EK, Elzer $\mathrm{PH}$. Buffalo, bush meat, and the zoonotic threat of brucellosis in Botswana. PLoS One. 2012;7:e32842.

31. Chase M: Dry season fixed-wing aerial survey of elephants and wildlife in Northern Botswana. Elephants without Borders, San Diego Zoological Society, Department of Wildlife and National Parks. Report 2011, pp. 138

32. Nijhof AM, Penzhorn BL, Lynen G, Mollel JO, Morkel P, Bekker CPJ, et al. Babesia bicornis sp. nov. and Theileria bicornis sp. nov.: tick-borne parasites associated with mortality in the black rhinoceros (Diceros bicornis). J Clin Microbiol. 2003;41:2249-54

33. Bekker CP, de Vos S, Taoufik A, Sparagano OA, Jongejan F. Simultaneous detection of Anaplasma and Ehrlichia species in ruminants and detection of Ehrlichia ruminantium in Amblyomma variegatum ticks by reverse line blot hybridization. Vet Microbiol. 2002:89:223-38

34. Nijhof AM, Pillay V, Steyl J, Prozesky L, Stoltzs WH, Lawrence JA, et al. Molecular characterization of the Theileria species associated with mortality in four species of African antelopes. J Clin Microbiol. 2005:43:5907-11.

35. He L, Feng H, Zhang W, Zhang Q, Fang R, Wang L, et al. Occurrence of Theileria and Babesia species in water buffalo (Bubalus babalis, Linneaus, 1758) in the Hubei province, South China. Vet Parasitol. 2011;186:490-6.

36. Pfitzer S, Oosthuizen MC, Bosman AM, Vorster I, Penzhorn BL. Tick-borne blood parasites in nyala (Tragelaphus angasii, Gray 1849) from KwaZuluNatal, South Africa. Vet Parasitol. 2011;176:126-31.

37. Lohr KF, Ross JPJ. Improvement of the indirect fluorescent antibody test for the diagnosis of diseases caused by intra-erythrocytic parasites. Z Tropenmed Parasitol. 1969;19:427.

38. Burridge MJ. Application of the indirect fluorescent antibody test in experimental East Coast fever (Theileria parva infection in cattle). Vet Res. 1971;12:338-41.

39. Billiouw M, Brandt J, Vercruysse J, Speybroeck N, Marcotty T, Mulumba M, et al. Evaluation of the indirect fluorescent antibody test as a diagnostic tool for East Coast fever in eastern Zambia. Vet Parasitol. 2005;127:189-98.

40. Cohen J. A coefficient of agreement for nominal scales. Educ Psychol Meas. 1960;20:37-46.

41. Tompkins DM, Dunn AM, Smith MJ, Telfer S. Wildlife diseases: from individuals to ecosystems. J Anim Ecol. 2011;80:19-38.
42. Anderson K, Ezenwa VO, Jolles AE. Tick infestation patterns in free ranging African buffalo (Syncerus caffer): effects of host innate immunity and niche segregation among tick species. Int J Parasitol Parasites Wildl. 2013;2:1-9.

43. Burridge MJ, Kimber CD. The indirect fluorescent antibody test for experimental East Coast fever (Theileria parva infection of cattle): evaluation of a cell culture schizont antigen. Res Vet Sci. 1972;13:451-5.

44. Fujisaki K, Kawazu S, Kamio T. The taxonomy of the bovine Theileria spp. Parasitology. 1994;10:31-3.

45. O'Sullivan MV, Zhou F, Sintchenko V, Kong F, Gilbert GL. Multiplex PCR and reverse line blot hybridization assay (mPCR/RLB). J Vis Exp. 2011;54:e2781

46. Young AS, Brown CG, Burridge MJ, Grootenhuis JG, Kanhai GK, Purnell RE, et al. The incidence of theilerial parasites in East African buffalo (Syncerus caffer). Tropenmed Parasitol. 1978;29:281-8.

47. Allsopp MT, Theron J, Coetzee ML, Dunsterville MT, Allsopp BA. The occurrence of Theileria and Cowdria parasites in African buffalo (Syncerus caffer) and their associated Amblyomma hebraeum ticks. Onderstepoort J Vet Res. 1999;66:245-9.

48. Bakheit MA, Latif AA. The innate resistance of Kenana cattle to tropical theileriosis (Theileria annulata infection) in the Sudan. Ann N Y Acad Sci. 2002;969:159-63.

49. Walker JG, Klein EY, Levin SA. Disease at the wildlife-lifestock interface: acaricide use on domestic cattle does not prevent transmission of a tick-borne pathogen with multiple hosts. Vet Parasitol. 2014;199:206-14.

50. Pienaar R, Potgieter FT, Latif AA, Thekisoe OMM, Mans BJ. Mixed Theileria infections in free-ranging buffalo herds: implications for diagnosing Theileria parva infections in Cape buffalo (Syncerus caffer). Parasitology. 2011;138:884-95.

51. Debeila EM: Occurrence of Anaplasma and Ehrlichia species in African buffalo (Syncerus caffer) in Kruger National Park and Hluhluwe-iMfolozi Park in South Africa. MSc dissertation. Department of Veterinary Tropical Diseases, University of Pretoria, Pretoria, South Africa; 2011

52. Oura CAL, Tait A, Asiimwea B, Lubega GW, Weir W. Haemoparasite prevalence and Theileria parva strain diversity in Cape buffalo (Syncerus caffer) in Uganda. Vet Parasitol. 2011;175:212-9.

53. Penzhorn BL. Babesiosis of wild carnivores and ungulates. Vet Parasitol. 2006;138(1-2):11-21

54. Musuka GN, Mafika M, Molathwa E, Nuru H. New distribution records of Amblyomma hebraeum in the sandveld region of Botswana: to the editor. J S Afr Vet Assoc. 2001;72:3.

55. Paine GD. Ticks (Acari: Ixodoidea) in Botswana. Bull Ent Res. 1982;72:1-16.

56. Walker JB, Mehlitz D, Jones GE: In Notes on the ticks of Botswana. GTZ, Eschborn: German Agency for Technical Cooperation, Ltd. 1978: 27, 33, $39-43,55,65$

57. Anonymous. The eradication of East Coast fever in South Africa. J S Afr Vet Ass. 1981;52:71-3.

58. Neitz WO. Corridor disease: a fatal form of bovine theileriosis encountered in Zululand. Bull Epizoot Dis Afr. 1955:3:121-3.

59. Olwoch JM, Reyers B, Engelbrecht FA, Erasmus BFN. Climate change and the tick-borne disease, Theileriosis (East Coast fever) in sub-Saharan Africa. J Arid Environments. 2008;72:108-20.

60. Caron A, Migue E, Gomo C, Makaya P, Pfukenyi D, Hove T, et al. Relationship between burden of infection in ungulate populations and wildlife/livestock interfaces. Epidemiol Infect. 2013;141:1522-35. 